\title{
New methods in ambulatory blood pressure monitoring: Interactive monitoring and detection of posture and movement patterns
}

\author{
Thomas PriLl \\ Central Institute of Mental Health, Mannheim, Germany \\ AND \\ JOCHEN FAHRENBERG \\ University of Freiburg, Freiburg, Germany
}

\begin{abstract}
Psychophysiological monitoring can be used to assess emotional reactivity in cardiovascular measures. Since blood pressure (BP) variability in daily life is primarily caused by physical activity, metabolic and nonmetabolic effects are confounded. A newly developed method of multiple accelerometry allows for the control of such unwanted variances by continuously detecting posture, general activity, and distinct movement patterns. Contingent on episodes of additional heart rate (AHR), an indicator of emotional reactivity, BP measurements can be triggered and participants prompted to enter their current mood in a handheld PC. To evaluate both new methods for BP research, we performed 24-h ambulatory monitoring with 40 normotensive student participants, an evaluation designed to include standard settings for a controlled comparison (library vs. cinema). Findings indicated that the group at the cinema showed higher values of AHR, but group differences in BP were not observed. On the whole, such multiple accelerometry and interactive monitoring appear to be useful methods in behavior research.
\end{abstract}

The methodology of ambulatory monitoring has progressed considerably in recent years. The methodology of monitoring has endured criticism, however, both because the acquisition of data is carried out in daily-life situations, under individually diverse conditions, and because the multiple effects are difficult to separate.

In medicine today, blood pressure (BP) monitoring and ECG are commonly used, routine methods for monitoring high-risk patients. Psychophysiological research, however, endeavors to achieve a more detailed analysis of BP reactions in order to answer such questions as which activities and what kind of emotional strain and mental load cause $\mathrm{BP}$ to rise? The unique psychophysiological approach to blood pressure behavior has had to overcome a large number of methodological difficulties. A large proportion of the variance of phasic BP changes is caused by changes in physical activity - that is, by adaptive changes in circulation to current metabolic demands. Separating the emotional and mental components from the metabolic component of heart rate change is difficult, since many different behaviors and activities are associated with changes in movement activity. Therefore, new methods of monitoring have been developed.

\section{Activity, Posture, and Movement Behavior}

Previous investigations used uniaxial accelerometer recordings or self-ratings of physical activity to assess posture and movement behavior, even though such effects have often been disregarded completely. However, the assessment of conditions in which participants are at rest versus walking or climbing stairs is essential to the psychophysiological investigation of cardiovascular change and energy expenditure under natural conditions. The precise detection of rest position and the detection of body rotation (e.g., whether a participant is sleeping on the left or right side) is important for a more precise evaluation of nightly blood pressure changes (Pickering, 1991).

The assessment of posture and movement, and the kinematic analysis of behavior in general, has profited greatly from the progress made in sensor technology and in advanced methods in signal analysis. Indeed, multichannel accelerometry has been evaluated in a number of ambulatory monitoring studies (e.g., Bussmann et al., 2001; Foerster \& Fahrenberg, 2000).

The detection of motion patterns can be improved by defining individual reference patterns for each posture and activity condition. This can be achieved by initial recording of the essential patterns under investigation. Multivariate analyses and pattern similarity coefficients can be used in conjunction with a standard protocol for the detection and labeling of an actual segment; that is, a discrete motion pattern is determined with reference to the standard protocol. A methods study has revealed that 13 different posture and activity conditions can be detected

T.Prill, thomas.prill@zi-mannheim.de or prill.thomas@web.de 
reliably, with only $3.2 \%$ misclassifications (Foerster \& Fahrenberg, 2000).

\section{New Strategies in Ambulatory Assessment}

New assessment strategies have been developed that apply principles of standardized psychological testing and of experimental design to ambulatory assessment.

Controlled (structured) assessment. Segments of standardized or semistandardized measurement are included that serve as a reference for inter- and intraindividual comparisons.

Interactive assessment. Contingent on changes of certain physiological parameters, a participant can be prompted by a beeper signal to record specific events, activities, or symptoms. The work of Myrtek on interactive monitoring of additional heart rate has been exemplary in this regard (Myrtek, 2004; Myrtek et al., 1988).

\section{Computer-Assisted Self-Reports}

Ambulatory assessment of subjective state or symptom reports by handheld PCs appears to have many advantages in comparison with the paper-and-pencil method: alarm functions for prompting participants at predefined intervals, and a built-in reminder signal; reliable timing of input; flexible layout of questions and response categories; branching of questions and tailor-made sequential or hierarchical strategies; concealment of previously recorded responses from the participants; reduction of retrospection bias; and convenience and ease of transfer of the data to a stationary PC for statistical analysis. One can generally assume a higher reliability from such assessments (Fahrenberg, Hüttner, \& Leonhart, 2001; H ufford, Shiffman, Paty, \& Stone, 2001).

\section{Additional Heart Rate (AHR) and the Freiburg Monitoring System (FMS)}

This innovative methodology consists of monitoring heart rate and physical activity in daily life and of the online partialing out of heart rate increases caused by physical activity. The remaining (additional) heart rate is assumed to indicate momentary emotional activation (or mental load) andwith some practical constraints - serves for the interactive assessment of changes in emotion. The recorder/analyzer is programmed to trigger a handheld $\mathrm{PC}$, which in turn signals the participant, with a beeper, to give a self-report on momentary activity, situation, and emotion. This occurs when the AHR exceeds a certain threshold, which is adjusted automatically to follow the general trend. Control periods are obtained by randomly interspersed trigger signals (Myrtek, 2004; Myrtek et al., 1988): The AHR detection algorithm was used and validated in a series of studies with many different samples and with around 1,300 participants.

The FMS (Myrtek, Foerster, \& Brügner, 2001) contains the essential components of this methodology: continuous recording of the ECG, calibrated multiple accelerometry, measurement of general activity, calculation of AHR in real time (online), and triggering via a handheld PC. The detection of changes in posture and movement patterns is conducted offline. The trigger signal may be used to initiate further devices - for example, a BP monitor. Thus, the
FMS was adapted for psychophysiological research in BP reactivity in daily life. This methodology was used in the present field study.

\section{Aims and Hypotheses}

The aim of this study was to evaluate two innovative methodologies in BP research: (1) continuous recording of physical activity, postures, and distinct motion patterns, and (2) interactive monitoring of AHR (measurements depending on the occurrence of an AHR). To this end, our 24-h BP monitoring design included a section of largely standardized activities (controlled monitoring). The first of the two groups viewed an emotional movie in the cinema, and the second group spent time quietly reading in the university library (reading room).

We hypothesized that heart rate and BP would be higher for feedbacks initiated by an AHR (true signal) than for randomly interspersed feedbacks without an AHR (random signal). In addition, higher self-ratings of emotional activation would be elicited at true than at random signals. Overall, we expected higher AHR, heart rate, and BP, as well as higher self-ratings of emotional activation, at the cinema.

\section{METHOD}

\section{Participants}

In this study, 52 male university students from several faculties were recruited as paid voluntary participants over a period of 8 months. All participants provided written informed consent prior to their participation. In all, 12 of the data sets were excluded because of artifacts, mainly caused by technical problems not directly associated with the research question. The participants were randomly assigned to one of the two equally sized groups (library or cinema). Nearly all of them had normal blood pressure (4 had mildly elevated BP at initial rest in the laboratory; that is, systolic BP $>139$ and/or diastolic BP $>89 \mathrm{mmHg}$.). The groups did not differ in physical features or in baseline measures of BP and heart rate (Table 1).

Table 1

Mean Comparisons Between the Two Groups (Library vs. Cinema) for the Physical Characteristics and the Baseline Measurements

\begin{tabular}{|c|c|c|c|c|c|c|}
\hline & \multicolumn{2}{|c|}{$\begin{array}{c}\text { Library } \\
(N=20)\end{array}$} & \multicolumn{2}{|c|}{$\begin{array}{c}\text { Cinema } \\
(N=20)\end{array}$} & \multirow[b]{2}{*}{$l$} & \multirow[b]{2}{*}{$p$} \\
\hline & $M$ & $\overline{S D}$ & $M$ & $\overline{S D}$ & & \\
\hline Age (years) & 25.1 & 3.8 & 24.5 & 3.6 & 0.47 & .64 \\
\hline Body weight $(\mathrm{kg})$ & 73.3 & 7.7 & 72.5 & 9.8 & 0.29 & .78 \\
\hline Body size $(\mathrm{cm})$ & 179.3 & 5.3 & 180.2 & 5.9 & -0.48 & .64 \\
\hline \multicolumn{7}{|c|}{ Heart Rate (bpm, ECG) } \\
\hline Night (lying) & 57.3 & 6.9 & 58.1 & 7.5 & -0.33 & .74 \\
\hline Laboratory (sitting) & 70.9 & 12.3 & 68.1 & 6.7 & 0.91 & .37 \\
\hline \multicolumn{7}{|c|}{ Systolic Blood Pressure (mmHg) } \\
\hline Night (lying) & 112.1 & 12.0 & 110.2 & 6.4 & 0.60 & .55 \\
\hline Laboratory (sitting) & 126.7 & 12.6 & 122.3 & 7.7 & 1.33 & .19 \\
\hline \multicolumn{7}{|c|}{ Diastolic Blood Pressure (mmHg) } \\
\hline Night (lying) & 60.0 & 8.2 & 60.2 & 4.6 & -0.06 & .95 \\
\hline Laboratory (sitting) & 76.9 & 9.6 & 75.6 & 6.9 & 0.46 & .65 \\
\hline
\end{tabular}

Note $-t$ tests are for independent samples. 


\section{Procedure}

The participants' premonitoring instruction and testing took place at the laboratory of the Psychophysiological Research Group, Freiburg. After electrodes and accelerometers were attached and checked, the BP cuff was applied and initial measurements were conducted. Afterward, subtypes of movement behavior were obtained to serve as reference conditions for the hierarchical classification of the posture and movement patterns (standard protocol). Finally, after further baseline measures, the participants were familiarized with the handling of the devices, and the 24-h monitoring began (the investigations of the different individual participants started at different points in time).

Participants belonging to the cinema group were instructed to view an emotional movie in the cinema (in one of four genres - action, thriller, melodrama, or horror-selected by the investigator), and the members of the library group were instructed to spend the time quietly at a certain place in the reading room of the university library. For the rest of the day, the participants' activities were not restricted, except for activities that would require extreme exertion or be unsafe with the monitoring equipment (e.g., taking a shower). However, all participants were asked to manually initiate a BP measurement on one occasion after climbing a stairway. During the recording period, all participants kept a log in which they recorded the period during which they were in the controlled setting (library or cinema) and the time at which they measured BP as requested, as well as their times of going to sleep and of other specific events.

The next day, after removing the devices, the participants filled out a questionnaire concerning general issues, such as reactivity phenomena and the unpleasantness of the study. After postmonitoring debriefing, the participants received their financial reward.

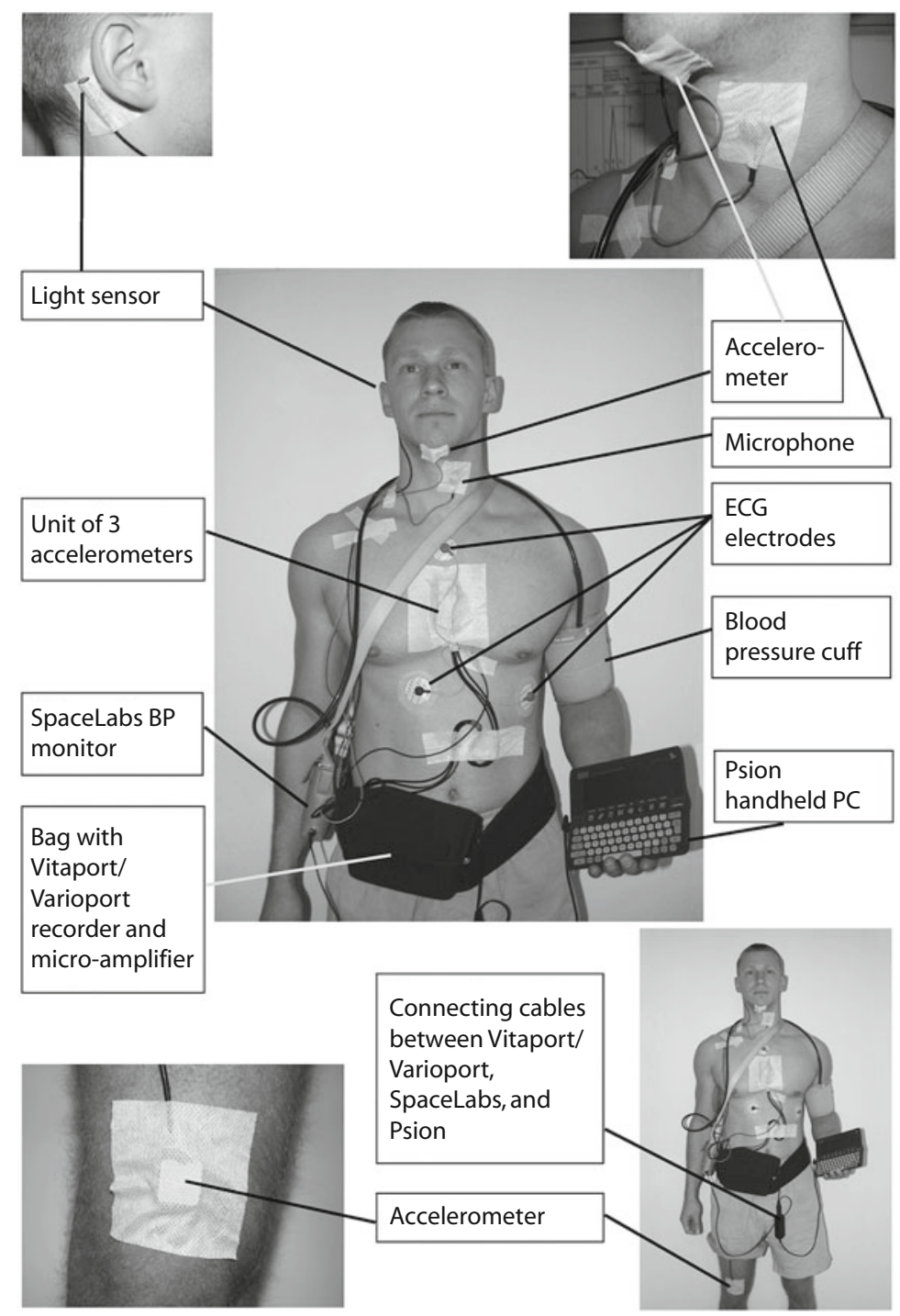

Figure 1. Placement of the ECG electrodes, accelerometers, and devices. 
Table 2

Devices Used, Variables, and the Allocation of the Channels

\begin{tabular}{|c|c|c|c|c|}
\hline Device & Channels & Placement & Variable & Software/Algorithm \\
\hline $\begin{array}{l}\text { Vitaport/Varioport } \\
\text { (recorder/analyzer; } \\
8 \text { channels) }\end{array}$ & $\begin{array}{l}\text { 1. ECG-F } \\
\text { 2. Accelerometer } \\
\text { 3. Accelerometer } \\
\text { 4. Accelerometer } \\
\text { 5. Accelerometer } \\
\text { 6. Accelerometer } \\
\text { 7. Light sensor } \\
\text { 8. Voice }\end{array}$ & $\begin{array}{l}\text { Nehb anterior (mod.) } \\
\text { Sternum (sagittal) } \\
\text { Sternum (transversal) } \\
\text { Sternum (vertical) } \\
\text { Right thigh (sagittal) } \\
\text { Chin (sagittal) } \\
\text { Processus mastoideus } \\
\text { Lateral at larynx }\end{array}$ & & $\begin{array}{l}\text { Special filtering } \\
\text { FMS algorithm for detection } \\
\text { of } 10 \text { subtypes of motion } \\
\text { patterns and postures }\end{array}$ \\
\hline Calculated variables: & & & $\begin{array}{l}\text { Physical activity } \\
\text { Heart rate (ECG) } \\
\text { Additional heart rate } \\
\text { Feedback (types) }\end{array}$ & $\begin{array}{l}\text { Index derived from } \\
\text { Channels 2, 3, 4, and } 5 \\
\text { Algorithm (FMS) } \\
\text { Algorithm (FMS) }\end{array}$ \\
\hline $\begin{array}{l}\text { SpaceLabs Model } 90207 \\
\quad \text { (blood pressure [BP] monitor) }\end{array}$ & & Upper arm (BP cuff) & $\begin{array}{l}\text { Systolic BP } \\
\text { Diastolic BP } \\
\text { Heart rate }\end{array}$ & Trigger by algorithm (FMS) \\
\hline $\begin{array}{l}\text { Psion Series 3a } \\
\quad \text { (handheld PC) }\end{array}$ & & & $\begin{array}{l}\text { Acoustic alarm } \\
\text { Self-reports }\end{array}$ & $\begin{array}{l}\text { Trigger by algorithm (FMS) } \\
\text { MONITOR for data acquisition }\end{array}$ \\
\hline
\end{tabular}

\section{Physiological Methods}

Figure 1 depicts the placement of devices, electrodes, and accelerometers. An overview of the channels and variables used is presented in Table 2 .

Electrocardiogram (ECG). The ECG was recorded via a chest lead in which the R-wave was optimized (modified Nehb anterior lead).

Calibrated multiple accelerometry. The accelerometers (IC Sensor, Model 3031) were piezoresistive and had wide bandwidth (i.e., DC and AC response), high sensitivity $(\sim 1 \mathrm{mV} / G$; standard range, $\pm 2 G)$, and a typical accuracy of $\pm 0.2 \%$. The frequency response was practically linear up to the kilohertz range. The accelerometers (supplied by Meditec Becker, Karlsruhe, Germany) were $20 \times$ $20 \times 2 \mathrm{~mm}$ in dimension and weighed $4 \mathrm{~g}$.

Each accelerometer was calibrated for a specific Vitaport/Varioport amplifier channel by measuring the signal under controlled inclination - that is, by rotating the accelerometer, providing a signal output corresponding to $+1 G$ and $-1 G$ (the gravitational constant). The DC output was zero when the sensitive axis was perpendicular to the gravitational axis. The recordings were obtained with a $32-\mathrm{Hz}$ sampling rate and low-pass filtering at $24 \mathrm{~Hz}$. A higher sampling rate was both unnecessary and, given the limited storage and battery power, undesirable. Physical activity was expressed as units (the signals were vectorial added and transformed in units by logarithmic amplification).

The accelerometers were positioned as follows:

Sternum. A unit of three uniaxial accelerometers was placed at the sternum about $5 \mathrm{~cm}$ below the jugulum, with the sensitive axes pointing in vertical, sagittal, and lateral directions - that is, in the $z-, x$-, and $y$-directions, respectively.

Thigh. Another accelerometer was placed on the frontal aspect of the right thigh, distal from $\mathrm{m}$. rectus femoris, about $5 \mathrm{~cm}$ above the patella, with the sensitive axis pointing in a sagittal direction.

The accelerometers were fixed to the skin with adhesive medical tape.
The standard protocol was performed in a fixed order, with each condition lasting at least $40 \mathrm{sec}$ : (1) sitting upright, (2) sitting leaning forward about $20^{\circ}$ from upright position, (3) sitting leaning backward about $-45^{\circ}$ from upright position, (4) standing, (5) lying back, (6) lying on the right side, (7) lying on the left side, (8) walking, (9) going upstairs, (10) going downstairs.

Blood pressure monitoring. The SpaceLabs (Model 90207) BP monitor was set to record ambulatory BP at intervals of $60 \mathrm{~min}$ during the 24-h monitoring period (including the night). Additional measurements associated with the feedback algorithm were restricted to daytime.

Further parameters. Further channels were used to continuously obtain data that were useful in the discrimination of settings and activities. For the recording of head movements (e.g., nodding), an accelerometer was placed below the chin. Speaking was recorded with a small electrodynamic microphone placed laterally at the larynx. The light sensor was a photodiode BPW mounted on the processus mastoideus. The microphone and the sensors were fixed with adhesive medical tape, and all signals were sampled at $32 \mathrm{~Hz}$.

Recorder/analyzer. Vitaport 2 or Varioport (Becker Meditec, Karlsruhe, Germany) were used for the multichannel recording. Vitaport 2 and Varioport are generalpurpose digital recorders/analyzers (32-bit microprocessor, $16 \mathrm{MHz}$ ) with minimized dimensions and power consumption designed for prolonged ambulatory recording. They weigh $700 \mathrm{~g}$ and $170 \mathrm{~g}$, respectively. The recorder was carried in a padded bag worn on a belt at the waist. Postprocessing was carried out with the Vitagraph software (Jain, Martens, Mutz, Weiß, \& Stephan, 1996) or with add-on analysis programs developed by the user.

\section{Self-Report Data Methods}

A handheld PC was used for recording self-reports. A Psion handheld computer (Series 3a, Psion PLC, London) and the MONITOR software were used (Fahrenberg et al., 2001). MONITOR is a flexible program with an exten- 
sive repertoire of features. In the present study, MONITOR displayed items regarding participants' whereabouts, companionship, their activities and current feelings (active/efficient, strenuous/burdensome situation, mentally tense, emotionally tense, excited/nervous, depressed, exhausted/sleepy, and distracted by answering the items). The participants were instructed in the use of the equipment and trained how to respond to questions presented on the handheld PC's display.

\section{Controlling and Timing of Data Acquisition}

The recorder/analyzer, Vitaport or Varioport, was connected by a cable to the BP monitor and the handheld PC. The feedback algorithm triggered automatic BP measurements and activated the handheld PC. BP measurement usually required about $40-50 \mathrm{sec}$, and the handheld PC was therefore activated $60 \mathrm{sec}$ after the BP monitor (the handheld PC questions concerned the point in time immediately prior to the $\mathrm{BP}$ measurement). The handheld PC and $\mathrm{BP}$ monitor were only triggered during the day. Prior to sleep, the participants were able to turn off the handheld $\mathrm{PC}$ - thus ensuring that both devices could not be triggered during sleep - but the recording of physiological variables, including the hourly BP measurements, continued without any interruption. Upon waking, the participants were requested to turn on the handheld $\mathrm{PC}$ again.

Additional heart rate. Calculation of episodes of additional heart rate took place online. Heart rate and physical activity during each minute were compared with the moving average for the previous $3 \mathrm{~min}$ in order to detect emotional events. An emotional event was assumed if the heart rate of a given minute was at least 3 beats $/ \mathrm{min}$ greater than the average of the previous minutes, with no or negligible increase in physical activity. If physical activity increased, the minimal additional heart rate used to indicate an emotional event increased as well. The algorithm for this procedure was adapted individually in order to optimize the detection of emotional events (for a detailed description, see Myrtek, 2004; Myrtek et al., 2001). The devices (BP monitor and handheld $\mathrm{PC}$ ) were triggered every 10-20 min, resulting in an average of four different measuring times per hour (after a feedback, there was no further feedback in the following $10 \mathrm{~min}$ ). The feedbacks were either "true" signals, indicating the occurrence of additional heart rate, or "random" signals, without additional heart rate. True and random signals were activated at an approximate ratio of 3:1. For interindividual comparisons of the AHR, offline calculations are used that were not affected by the adaptive procedures for each individual. In order to achieve integer numbers, AHR was multiplied by 10 .

Validity of the AHR algorithm in the present study. To ensure that physical activity reflects metabolic demand, there must be a strong correlation between physical activity, as measured by the accelerometers, and heart rate. To make certain that AHR is not caused by metabolic demand, there should be no correlation between AHR and physical activity (Myrtek, 2004). As expected, the (pooled) within-subjects correlation was high between activity and heart rate $(r=.74)$, low between AHR and activity $(r=$
$.03)$, and low between AHR and heart rate $(r=.18)$. Altogether, these correlations indicate the proper operation of the algorithm.

\section{Data Analysis}

Raw-signal data analysis and segmentation. The continuous physiological recordings were evaluated in several steps with the software system FMS (Myrtek et al., 2001), which includes SAS macros for a number of tasks: the parameterization of biological signals, visual control of artifacts, offline calculation of AHR, hierarchical classification of the posture and movement patterns, and the integration of data from different sources. The parameterization provided averages for each physiological variable, with a time resolution of $60 \mathrm{sec}$.

Segmentation into day and night segments was performed on the basis of the information given by the participants, and the segmentation was modified according to the records for movement activity and room brightness. The primary means of segmentation was the kinematic detection and analysis of posture and of movement patterns (with reference to the individual standard protocol). Some definitions were necessary in order to account for method-dependent time lags. The BP values of different postures and motion patterns were compared in the following way: The BP values were considered in relation to the posture and movement pattern in the minute preceding the measurement, because participants frequently discontinued a movement activity and rested so as not to disturb the BP measurement. Thus, the BP values largely reflect the activity prior to the measurement. In order to compare true and random signals, BP values were similarly related to conditions in the minute preceding the measurement, because the minute preceding BP measurement was used to calculate the corresponding AHR.

The library and cinema settings were of particular interest in this study. Here again the segmentation was based on the details provided by the participants and modified using the records of movement activity and posture. Moreover, the data from the light sensor was used to exactly detect the setting of the cinema. The essential condition for both settings was sitting position. Once these settings had been determined, only data from the sitting position were analyzed. This condition was also essential for the comparison of true versus random signals.

Statistical data analysis. The statistical analysis comprised descriptive statistics, $\chi^{2}$ tests, group comparisons through $t$ tests for independent samples, and a segment comparison using $t$ tests for dependent samples. A $p$ value of .05 was considered a statistically significant difference.

\section{RESULTS}

In the course of monitoring, an average of 1,205 valid minutes of recording were obtained from each participant. An average of 36 self-reports (via handheld PC) and 30 $\mathrm{BP}$ values following a feedback signal were recorded for each participant. The discrepancy in numbers between BP readings and self-reports can be explained by occasional 
artifacts in BP measurement. An average of 7 BP measurements and 7 self-reports were assessed for the controlled library/cinema settings (in sitting position).

\section{Comparison of the Signal Types in the 24-h Segment}

The hypothesis that a higher heart rate would be measured for true signals than for random signals in the sitting position was confirmed (difference $=4.7 \mathrm{bpm} ; t(39)=$ $5.23, p<.001$, effect size $d=0.45$ ). Contrary to our expectation, however, there was no significant increase in $\mathrm{BP}$ values for true signals (see Table 3). Comparisons between signal types revealed no differences in means and standard deviations related to different self-report items.

\section{Settings (Library vs. Cinema)}

Physiological parameters. As expected, in comparison with the library segment (total), the cinema segment (total) showed higher values of AHR (calculated offline; difference $=1.0 ; t(38)=2.56, p=.015, d=0.81)$. No group differences were evident, however, in the other cardiovascular parameters (heart rate or BP). Furthermore, there were no differences between the two groups in BP level- that is, in change scores relating to averages of nightly BP measures (lying position). But there were group differences with respect to the activity index, the average of which was higher in the library group (difference $=2.6 ; t(38)=4.68, p<.001, d=1.48)$. See the comparisons of the physiological parameters (library vs. cinema) in Figure 2.

Self-report data (handheld PC). In comparison with the group in the library, participants in the cinema scored higher for items of emotional activation; this confirmed the hypothesis (see Figure 3). In the cinema, the participants felt emotionally more tense (difference $=1.0$; $t(38)=3.17, p=.003, d=1.00$ ) and more nervous (difference $=1.0 ; t(38)=3.16, p=.003, d=1.00)$. Furthermore, in the cinema condition, participants felt more strongly distracted by having to answer the items.

\section{Further Comparisons}

Segment comparisons (day vs. night, resting vs. moving). Besides the comparison for the library versus

Table 3

Mean Comparisons Between Signal Minutes With Occurrence of an Additional Heart Rate (True Signals) and Minutes Without an Additional Heart Rate (Random Signals) for Physiological Data

\begin{tabular}{|c|c|c|c|c|c|c|}
\hline \multirow[b]{2}{*}{ Measure } & \multicolumn{2}{|c|}{$\begin{array}{c}\text { True } \\
\text { Signals }\end{array}$} & \multicolumn{2}{|c|}{$\begin{array}{l}\text { Random } \\
\text { Signals }\end{array}$} & \multirow[b]{2}{*}{$t$} & \multirow[b]{2}{*}{$p$} \\
\hline & $M$ & $S D$ & $M$ & $S D$ & & \\
\hline Systolic BP (mmHg) & 128.0 & 9.5 & 127.1 & 9.0 & 1.08 & .29 \\
\hline Diastolic BP (mmHg) & 76.7 & 7.7 & 76.6 & 7.6 & 0.26 & .80 \\
\hline Activity (units) & 12.3 & 3.4 & 11.8 & 6.8 & 0.42 & .68 \\
\hline Heart rate (bpm, ECG) & 79.7 & 9.0 & 75.0 & 11.6 & 5.23 & $<.00$ \\
\hline $\begin{array}{l}\text { Additional heart rate } \\
\text { (units, offline) }\end{array}$ & 13.4 & 2.7 & 0.3 & 1.0 & 29.69 & $<.00$ \\
\hline
\end{tabular}

Note-Comparisons are restricted to measures while in sitting position (without bicycling). $t$ test results are for dependent samples. $N=$ 39. BP, blood pressure. the cinema, Figure 2 also contains comparisons for day versus night and resting versus moving ("resting" includes all lying and sitting positions, as well as standing; "moving" includes walking, going up- and downstairs, and bicycle riding). Figure 3 also contains comparisons regarding emotional activation for resting versus moving and for the day segment.

Comparison of categories of posture and distinct motion patterns. Figure 4 depicts the differences between the categories of posture and distinct motion patterns with regard to BP and heart rate. An interesting comparison includes the three lying positions (lying back, on the left side, and on the right side of the body). Only right-handed participants, with the BP cuff mounted on their left arm, were considered. Lower blood pressure values were evident when lying on the right side of the body because the relative height of the cuff deviated from the level of the heart (difference: for systolic BP, $11 \mathrm{mmHg}$; for diastolic BP, $7 \mathrm{mmHg}$ ).

\section{Participants' Evaluation}

The participants completed a questionnaire so that we could evaluate their acceptance and responses to this particular method. Only 2 out of 11 items revealed significant differences between the library and cinema groups: The library group was more inclined to rate the investigation as interesting (5.5 on a 7-point scale, as compared with $4.7 ; t=2.3, p=.026$ ) and expressed greater willingness to take part in another study of this kind $[15$ participants stated yes and 5 maybe, as compared with 6 yes and 12 maybe from the cinema group; $\left.\chi^{2}(2)=8.74, p=.013\right]$. The participants were asked whether their behavior was influenced by the presence and use of the monitoring methodology and whether self-reports (via the handheld PC) were prompted too frequently. The participants responded on average with 3.4 and 4.4, respectively, on a 7-point rating scale $(1=$ not at all, $7=$ totally $)$.

\section{DISCUSSION}

The general aim of this investigation was to assess new strategies of BP monitoring under everyday conditions: (1) the continuous recording of physical activity, posture, and distinct motion patterns, and (2) interactive monitoring of additional heart rate. The advantages of calibrated multiple accelerometry in the detection of posture and motion during ambulatory monitoring were clearly demonstrated in this investigation. This methodology permits higher precision in segmentation (and standardization of the conditions for testing) and supports the evaluation of BP measures.

Depending on activities or settings, the segmentation of individual records may be difficult in some instances. In this case, the additional variables that we recorded proved useful. The light sensor allowed for more reliable discrimination between the cinema and other settings and between the day and night segments. Speech activity (voice) and increased head movements (nodding) were important sources of information for the determination of social interaction. The measurement of ambient parameters, such 

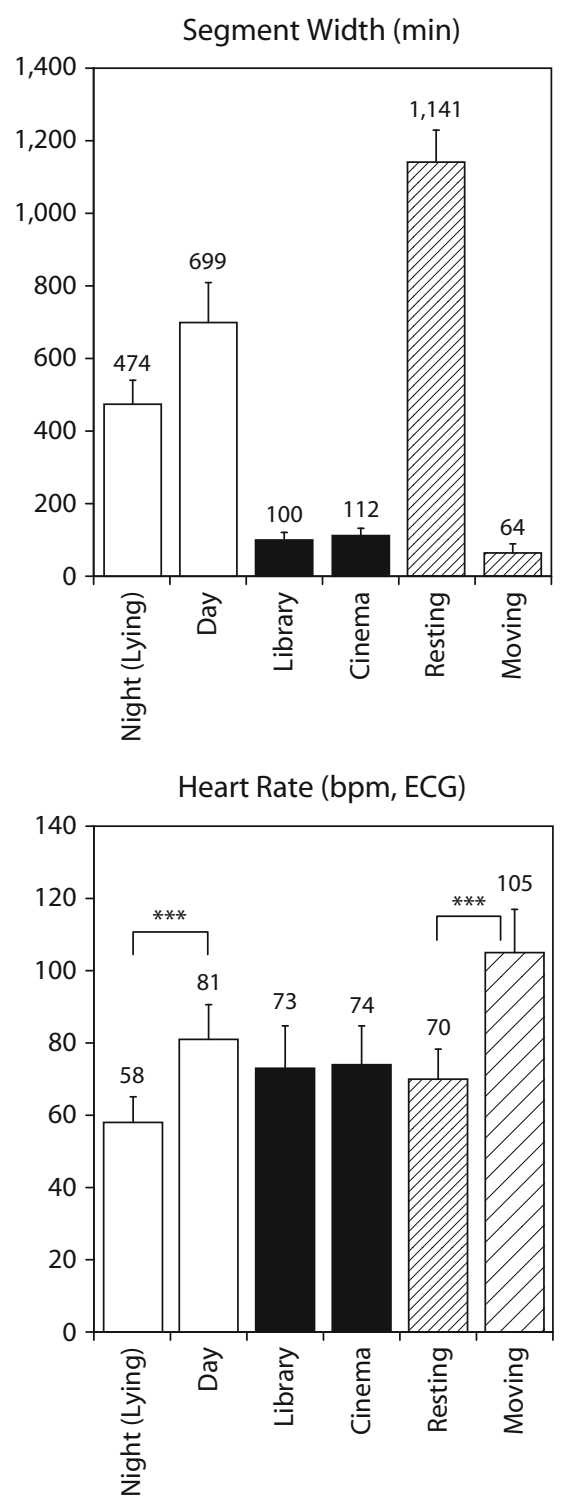

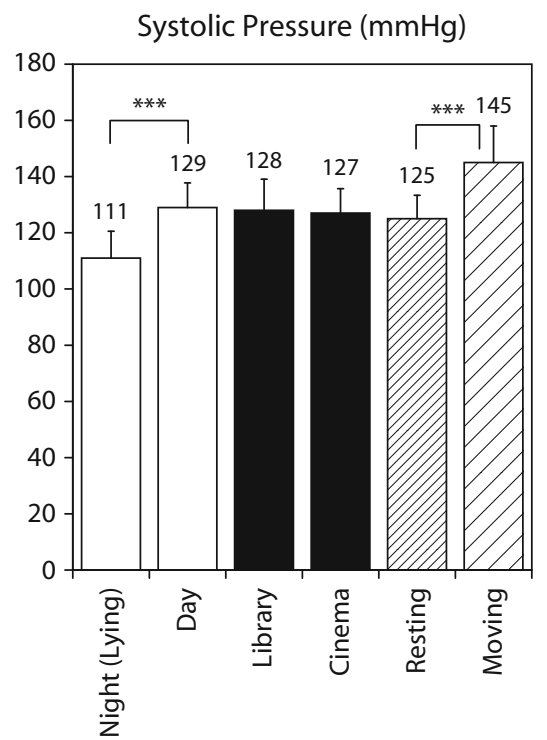

Activity (units)

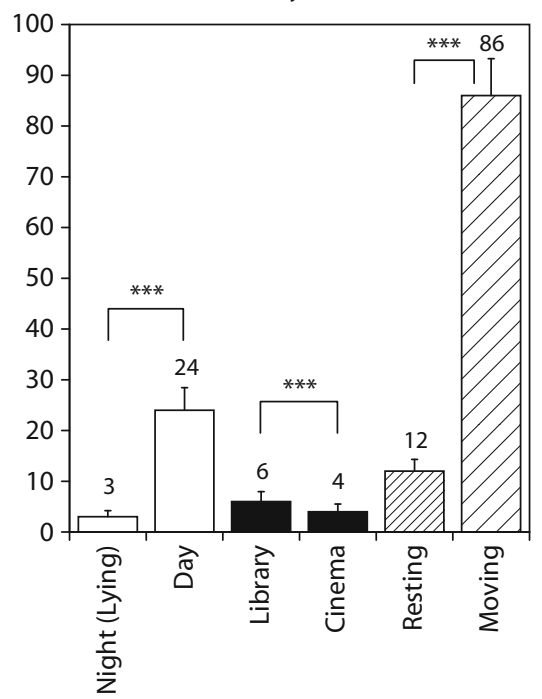

Diastolic Pressure $(\mathrm{mmHg})$

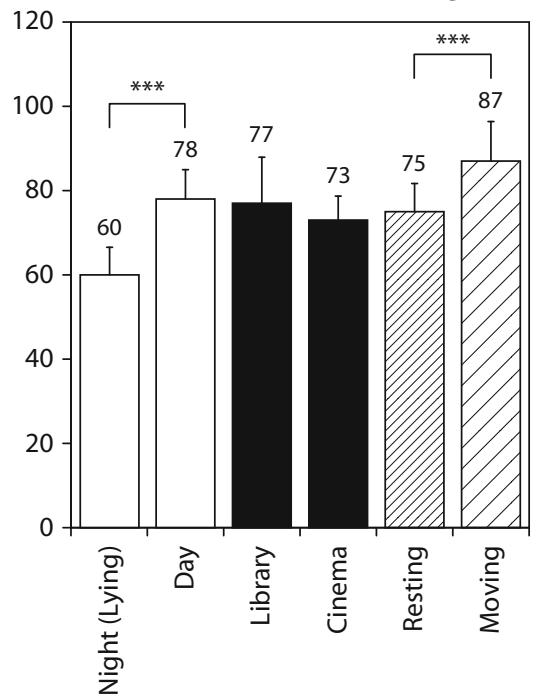

Additional Heart Rate (units)

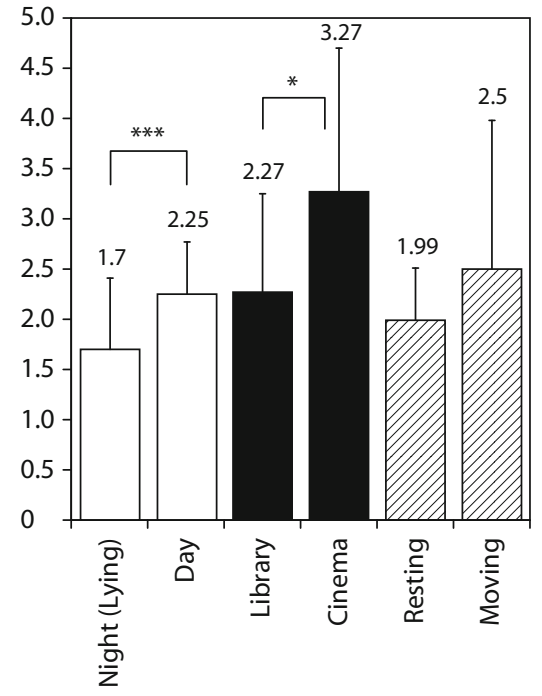

Figure 2. Mean comparisons of the physiological variables between the paired segments night/day (general segments), library/cinema (sitting position), and resting/moving. Means and standard deviations are included, as well as results from a $t$ test for the library versus cinema comparison $(N=20)$ and from a $t$ test for dependent samples (BP) for the comparisons night versus day and resting versus moving $\left(N=40\right.$ and $N=38$, respectively). $\quad{ }^{*} p<.05$. ${ }^{* * * *} p<.001$.

as noise level, temperature, and barometric pressure (indicating height), and the behavioral correlates of such data should be further evaluated in multichannel recordings (see Fahrenberg, Leonhart, \& Foerster, 2002).

The expected differences in degree of emotional activation between the settings were reflected in the self-report data. Participants in the cinema group were emotionally tenser and more nervous. Accordingly, the findings revealed that the cinema condition elicited a higher AHR. Corresponding to our expectation, "true" feedback signals were associated with a higher heart rate than were "random" feedback signals.

Occurrence of AHR was not associated with higher BP readings in this study. This finding seems to suggest that the AHR methodology is not suited to detection of emo- tional BP changes in ambulatory monitoring. However, several issues were involved.

Viewing emotional movies did not elicit the expected significant rise in BP. It appears that such reactions can be elicited only by movies with extremely provocative or distressing content (Myrtek, 2004). Obviously, substantial $\mathrm{BP}$ reactivity is required to test whether AHR and rises in $\mathrm{BP}$ occur concurrently. In the present investigation, the BP measurement was triggered following the detection of an AHR; that is, the measuring process resulted in BP values being available with a time lag of about $1 \mathrm{~min}$. It can be assumed that part of the dynamics were already lost before the BP measuring process was complete.

Furthermore, it is debatable whether phasic reactions in heart rate and BP are highly synchronized. In a methods 


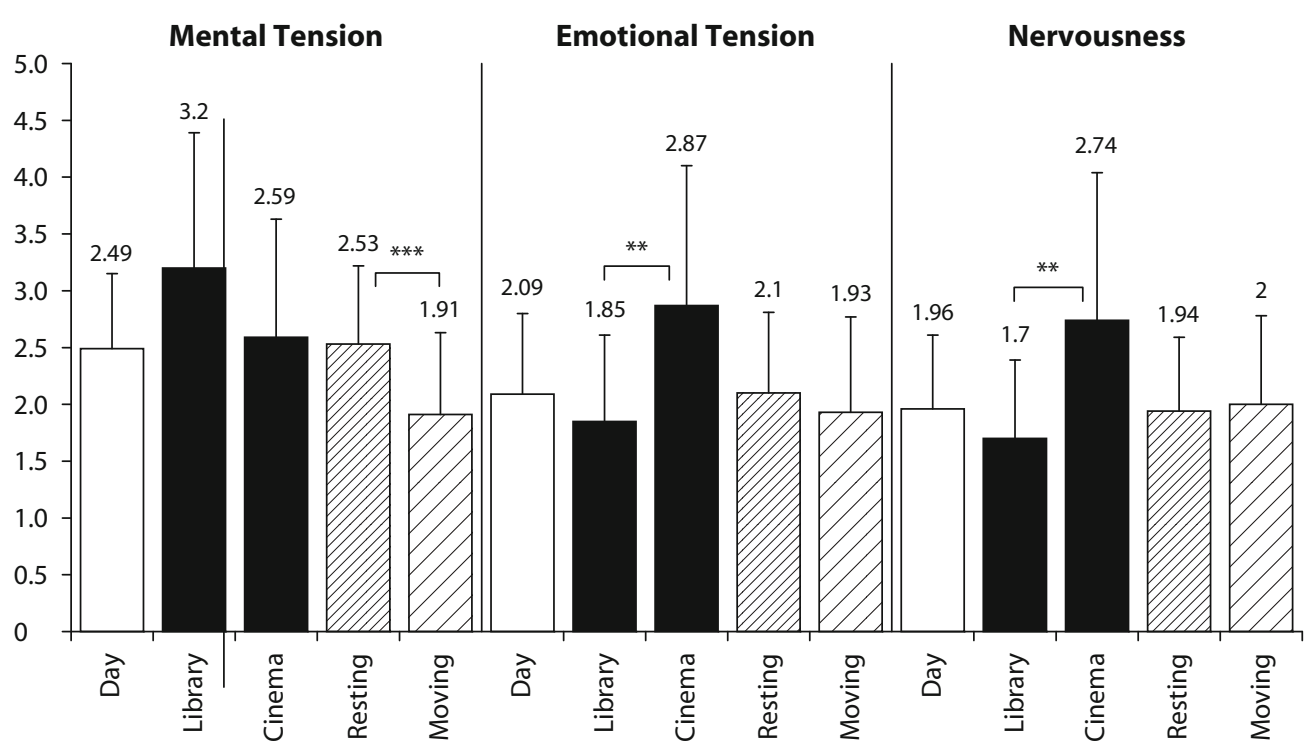

Figure 3. Mean comparisons of self-ratings (emotional activation) between the paired segments library/ cinema (sitting position) and resting/moving, referring to the day segment. Means and standard deviations are provided for the graduated answer choices $(1=$ not at all, $7=$ totally $)$, as well as the results of a $t$ test for the comparison library versus cinema $(N=20)$ and a $t$ test for dependent samples for the comparison resting versus moving $(N=40) .{ }^{* *} p<.01 .{ }^{* * *} p<.001$.

study, heart rate and finger BP were recorded by use of a Portapres device (BMI-TNO, Amsterdam) in 10 hypertensive patients during an interview, thus providing for appropriate variances. On average, 110 segments of 30- sec duration were used. Within-subjects correlation coefficients for systolic BP and heart rate ranged from .34 to .61 ; that is, the correlations were positive in each case, but from small to medium in size. Although the precision and

\section{Blood pressure and heart rate of distinct motion patterns and postures}
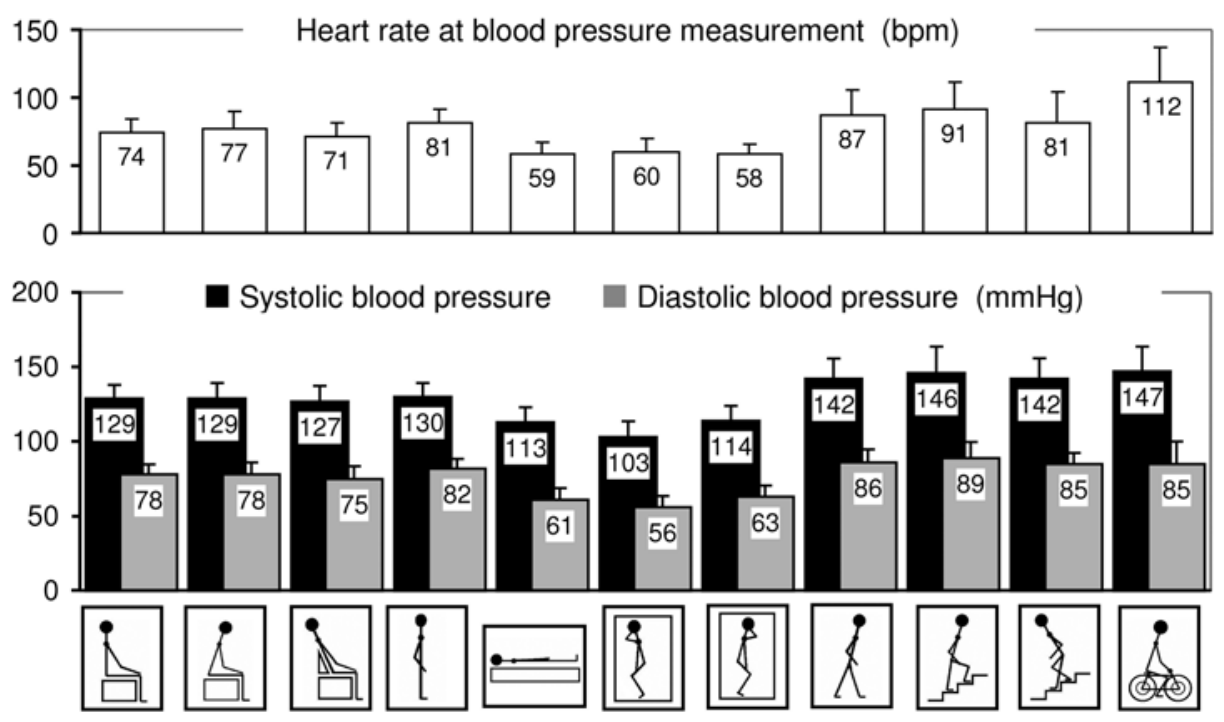

Figure 4. Blood pressure (BP) and heart rate (from a BP monitor) associated with distinct motion patterns and postures (means and standard deviations). The categories are, from left to right: sitting upright $(N=40)$; sitting leaning forward $(N=35)$; sitting leaning back $(N=37)$; $\operatorname{standing}(N=$ 40); lying on the back ( $N=32$, right-handers only); lying on the right side $(N=25$, right-handers only); lying on the left side $(N=25$, right-handers only); walking $(N=29)$; going upstairs $(N=$ 25); going downstairs $(N=7)$; cycling $(N=8)$. The classification is based on individual reference patterns according to Foerster's algorithm, with the exception of cycling, which was reported by participants and given a plausibility check by means of activity and posture. 
accuracy of finger BP measurement can be questioned, the findings indicate that the covariation of both cardiovascular parameters is far from perfect.

For this reason, we adapted the basic algorithm in order to define minutes of additional systolic BP in continuous recordings of finger BP (Portapres), and likewise, to trigger a handheld PC to obtain self-reports. In a feasibility study with 5 participants, again in the cinema setting, the new algorithm was tested successfully (Fahrenberg \& Foerster, 2002). At present, this is a demanding methodology not easily applied in larger studies.

With respect to the AHR algorithm, the present study showed that the algorithm's validity has certain limitations in specific situations. In the transition from movement on a level path to ascending a flight of steps, the accelerometers might not register an increase in movement activity. We found that the algorithm wrongly interpreted the rise in heart frequency inevitably associated with an increase in movement intensity as crossing the minimal threshold for AHR. This interpretive error can also arise if heavy objects are carried. The validity of the online calculation of an episode of AHR should be improved by taking the discrimination between walking and climbing into account, which could be achieved by incorporating a highly sensitive barometric sensor that measures step amplitude and differences in height.

Recently, there has been much progress in the methodology of ambulatory monitoring and assessment (see Fahrenberg, 2006; Fahrenberg \& Myrtek, 1996, 2001; see also the site of the European Network for Ambulatory Assessment, www.ambulatory-assessment.org). Basically, the present study proposes new applications and provides corresponding evidence for the use of the FMS methodology, which includes calibrated multiple accelerometry and the AHR algorithm for detection of nonmetabolic additional heart rate. Thus, interactive and controlled monitoring encourages new assessment strategies in behavior research.

\section{AUTHOR NOTE}

This research was supported in part by a grant from the Deutsche Forschungsgemeinschaft. We thank Marcus Cheetham for improving the English. Correspondence relating to this article may be sent to T. Prill, Central Institute of Mental Health, Department of Psychosomatic Medicine and Psychotherapy, Postfach 1221 20, 68072 Mannheim, Germany (e-mail: thomas.prill@zi-mannheim.de or prill.thomas@web.de).

\section{REFERENCES}

Bussmann, J. B. J., Martens, W. L. J., Tulen, J. H. M., Schasfoort, F. C., van den Berg-Emons, H. J. G., \& Stam, H. J. (2001). Measuring daily behavior using ambulatory accelerometry: The Activity Monitor. Behavior Research Methods, Instruments, \& Computers, 33, 349-356.
FAHRENBerg, J. (2006). Assessment inn daily life: A review of computerassisted methodologies and applications in psychology and psychophysiology, years 2000-2005. Retrieved August 12, 2007, from www .ambulatory-assessment.org.

FAHRENBERG, J., \& Foerster, F. (2002). Kontrolliertes und interaktives Blutdruck-Monitoring: Neue Strategien und Ergebnisse [Controlled and interactive blood pressure monitoring: New strategies and results] (Research Report No. 155). Freiburg: Albert-Ludwigs-Universität, Department of Psychology.

Fahrenberg, J., Hüttner, P., \& Leonhart, R. (2001). Psychological assessment in everyday life by hand-held PC: Applications of MONITOR. In J. Fahrenberg \& M. Myrtek (Eds.), Progress in ambulatory assessment: Computer-assisted psychological and psychophysiological methods in monitoring and field studies (pp. 93-112). Seattle: Hogrefe \& Huber.

Fahrenberg, J., Leonhart, R., \& Foerster, F. (2002). Alltagsnahe Psychologie: Datenerhebung im Feld mit hand-held PC und physiologischem Mess-System [Psychology of daily life: Data acquisition in the field with handheld PC and physiological recording systems]. Bern: Huber.

FAhrenberG, J., \& MYrTeK, M. (Eds.) (1996). Ambulatory assessment: Computer-assisted psychological and psychophysiological methods in monitoring and field studies. Seattle: Hogrefe \& Huber.

Fahrenberg, J., \& MyrteK, M. (EDS.) (2001). Progress in ambulatory assessment: Computer-assisted psychological and psychophysiological methods in monitoring and field studies. Seattle: Hogrefe \& Huber.

Foerster, F., \& FAHREnBerG, J. (2000). Motion pattern and posture: Correctly assessed by calibrated accelerometers. Behavior Research Methods, Instruments, \& Computers, 32, 450-457.

Hufford, M. R., Shiffman, S., Paty, J., \& Stone, A. A. (2001). Electronic momentary assessment: Real-world, real-time measurement of patient experience. In J. Fahrenberg \& M. Myrtek (Eds.), Progress in ambulatory assessment: Computer-assisted psychological and psychophysiological methods in monitoring and field studies (pp. 69-92). Seattle: Hogrefe \& Huber.

Jain, A., Martens, W. L. J., Mutz, G., Weiß, R. K., \& Stephan, E. (1996). Towards a comprehensive technology for recording and analysis of multiple physiological parameters within their behavioral and environmental context. In J. Fahrenberg \& M. Myrtek (Eds.), Ambulatory assessment: Computer-assisted psychological and psychophysiological methods in monitoring and field studies (pp. 215-235). Seattle: Hogrefe \& Huber.

MyrteK, M. (ED.) (2004). Heart and emotion: Ambulatory monitoring studies in everyday life. Seattle: Hogrefe \& Huber.

Myrtek, M., Brügner, G., Fichtler, A., König, K., MÜller, W., Foerster, F., \& HöppNer, V. (1988). Detection of emotionally induced ECG changes and their behavioural correlates: A new method for ambulatory monitoring. European Heart Journal, 9(Suppl.), $55-60$.

Myrtek, M., Foerster, F., \& Brügner, G. (2001). Freiburger Monitoring System (FMS): Ein Daten-Aufnahme-und Auswertungs-System für Untersuchungen im Alltag. Emotionale Beanspruchung, Körperlage, Bewegung, EKG - Subjektives Befinden - Verhalten [Freiburg monitoring system (FMS): A data recording and analysis system for studies in everyday life. Emotional stress, body position, movement, electrocardiography, subjective state, and behavior]. Frankfurt: Peter Lang.

Pickering, T. G. (1991). Ambulatory monitoring and blood pressure variability. London: Science Press.

(Manuscript received July 14, 2005; revision accepted for publication March 21, 2006.) 